\title{
PROGRAMAS SOCIALES Y REDUCCIÓN DE LA OBESIDAD EN EL PERÚ: REFLEXIONES DESDE LA INVESTIGACIÓN
}

\author{
Francisco Diez-Canseco ${ }^{1, a}$, Lorena Saavedra-Garcia ${ }^{1, b, c}$
}

\begin{abstract}
RESUMEN
En las últimas décadas, el sobrepeso y la obesidad han aumentado de forma acelerada en los países de ingresos medios y bajos, una situación que consolida a las enfermedades crónicas no transmisibles (ENT) como una de las más importantes causas de mortalidad y discapacidad a nivel global. Actualmente, uno de cada dos peruanos mayores de 15 años tiene exceso de peso y uno de cada cinco sufre de obesidad, una situación que está en continuo crecimiento y afecta cada vez más intensamente a los que viven en condiciones de pobreza, quienes, con frecuencia, son beneficiarios de programas de complementación alimentaria, dirigidos a enfrentar la inseguridad alimentaria y la desnutrición. Existe globalmente una urgente necesidad de encontrar políticas y programas que permitan enfrentar el problema de la obesidad a nivel poblacional, una tarea todavía pendiente. En este artículo revisamos la situación actual de la epidemia de sobrepeso y obesidad en el Perú y el mundo, sus consecuencias y determinantes más importantes, con énfasis en el acceso y disponibilidad de alimentos; describimos los programas de complementación alimentaria más importantes y sintetizamos información de algunas investigaciones e intervenciones para reflexionar sobre cómo sus hallazgos podrían servir para que los programas sociales sean una como plataforma para reducir la obesidad y prevenir las ENT en el Perú.
\end{abstract}

Palabras Clave: Obesidad; Programas de gobierno; Asistencia alimentaria; Perú (fuente: DeCS BIREME).

\section{SOCIAL PROGRAMS AND REDUCING OBESITY IN PERU: REFLECTIONS FROM THE RESEARCH}

\begin{abstract}
In recent decades, overweight or obesity have increased dramatically in middle- and low-income countries; a situation which consolidates chronic non-communicable diseases (NCD) as one of the leading causes of mortality and disability worldwide. Currently, half the people in Peru over the age of 15 years are overweight, and one fifth suffer from obesity. The situation is worsening and increasingly affects people in poverty, who frequently benefit from food supplement programs designed to combat food insecurity and malnutrition. There is an urgent worldwide need to find policies and programs that help fight the problem of obesity at the population level, a task that is still pending. In this article, we review the current epidemic of overweight and obesity in Peru and the world and its most significant consequences and causes, with an emphasis on access to and availability of foods. We describe the largest food supplement programs and synthesize the research on interventions in order to reflect on how their findings might help social programs work as a platform to reduce obesity and prevent NCD in Peru.
\end{abstract}

Key words: Obesity; Government programs; Food assistance; Peru (source: MeSH NLM).

\section{INTRODUCCIÓN}

La existencia de una epidemia de obesidad mundial, una idea que hace algunas décadas se consideraba una herejía ${ }^{(1)}$, es actualmente una realidad innegable. EI sobrepeso y la obesidad han aumentado vertiginosamente en países de ingresos medios y bajos ${ }^{(2)}$ como el Perú, extendiéndose entre los pobres, aquellos que tienen dificultades para lograr una nutrición adecuada y que en nuestro país suelen ser beneficiarios de programas sociales de complementación alimentaria.
En la primera parte de este artículo revisamos las cifras de la epidemia de sobrepeso y obesidad en el Perú y el mundo, sus consecuencias y determinantes más importantes, con énfasis en el acceso y la disponibilidad de alimentos. En la segunda parte repasamos los programas de complementación alimentaria más importantes y resumimos algunas investigaciones e intervenciones para luego reflexionar sobre la posibilidad de que los programas sociales sirvan como una plataforma para reducir la obesidad y prevenir las enfermedades crónicas no transmisibles (ENT) entre los ciudadanos más pobres del Perú.

\footnotetext{
CRONICAS Centro de Excelencia en Enfermedades Crónicas, Universidad Peruana Cayetano Heredia. Lima, Perú.

a Psicólogo, magíster en Salud Pública; ${ }^{\mathrm{b}}$ licenciada en Nutrición; ${ }^{\mathrm{c}}$ magíster en Alimentación, Nutrición y Metabolismo Recibido: 10/01/2017 Aprobado: 25/01/2017 En línea: 23/03/2017
} 


\section{MAGNITUDES, TENDENCIAS E IMPACTO EN LA SALUD DEL SOBREPESO Y LA OBESIDAD EN EL PERÚ Y EL MUNDO}

Entre 1980 y 2013, el número de personas con sobrepeso y obesidad aumentó en más del doble, pues pasó de 921 millones a 2,1 mil millones (2), alcanzando así a más de un tercio de la población mundial. Los datos disponibles revelan un aumento rápido y generalizado de la obesidad. Sin embargo, mientras las cifras de los países desarrollados revelarían que este aumento se ha atenuado en la última década, las cifras de países en desarrollo, donde viven dos de cada tres personas con obesidad, sugieren que esta seguirá aumentando ${ }^{(1,3)}$.

El Perú no ha estado en ajeno a estos cambios. Por el contrario, la información disponible muestra una alta prevalencia de sobrepeso y obesidad, y un aumento vertiginoso de estas condiciones en la última década. La Encuesta Demográfica y de Salud Familiar (ENDES) del año $2015^{(4)}$ revela que el índice de masa corporal (IMC) promedio de la población de 15 años a más es de $26,2 \mathrm{~kg} / \mathrm{m}^{2}$, y que uno de cada dos peruanos de 15 años o más tiene sobrepeso $(35,5 \%)$ u obesidad $(17,8 \%)$.

La Tabla 1, elaborada con información de la Encuesta Nacional de Hogares (ENAHO) de 2007-08 y 2013-14, es reveladora y preocupante, por la magnitud conjunta de sobrepeso y obesidad, y su notable incremento en todos los grupos de edad en un periodo tan corto.

Especialmente relevante para una reflexión sobre el rol de los programas sociales es el significativo y acelerado

Tabla 1. Prevalencias de sobrepeso y obesidad por grupos de población en el Perú, durante el periodo 2007-2008 y 2013-2014

\begin{tabular}{lccc}
\hline $\begin{array}{l}\text { Grupo de } \\
\text { población o de } \\
\text { edad }\end{array}$ & $\begin{array}{c}\text { Prevalencia de } \\
\text { sobrepeso y obesidad } \\
\text { (\%) }\end{array}$ & $\begin{array}{c}\text { \% de } \\
\text { incremento } \\
\mathbf{2 0 0 7 - 0 8 ~ a ~} \\
\mathbf{2 0 1 3 - 1 4}\end{array}$ \\
\cline { 2 - 3 } $\begin{array}{c}\text { Año 2007- } \\
\mathbf{0 8}\end{array}$ & $\begin{array}{c}\text { Año 2013- } \\
\mathbf{1 4}\end{array}$ & \\
\hline De 5 a 9 años & 19,4 & 32,3 & 66,5 \\
\hline De 10 a 19 años & 17,8 & 26,0 & 46,1 \\
\hline De 20 a 29 años & 33,9 & 45,0 & 32,7 \\
\hline De 30 a 59 años & 55,7 & 69,9 & 25,5 \\
\hline De 60 a más & 24,8 & 33,1 & 33,5 \\
\hline $\begin{array}{l}\text { Mujeres en edad } \\
\text { fértil (15-49 años) }\end{array}$ & 49,0 & 58,2 & 18,8 \\
\hline
\end{tabular}

Fuente: Observatorio del Estudio del Sobrepeso y Obesidad. Elaboración propia ${ }^{(5)}$ aumento de la obesidad en las mujeres en edad fértil del quintil inferior y del segundo quintil de riqueza, en los que la obesidad, entre 2010 y 2014, según la ENDES, pasó de 7,5 a $13,2 \%$ y de 13,2 a $21,8 \%$, respectivamente ${ }^{(5)}$.

Habitualmente, las cifras de sobrepeso y, más claramente, las de obesidad en el Perú, son más altas en mujeres que en varones, en zonas urbanas que en rurales, así como en las poblaciones de la costa en comparación con las de la sierra y la selva ${ }^{(4)}$.

Toda esta información no solo deja en claro que existe una alarmante cantidad de personas con sobrepeso u obesidad en el Perú, y que estas condiciones aumentan rápidamente, sino que, además, nos invita a pensar en la importancia de comprender las variaciones que existen en distintos grupos poblacionales, en las razones de estas diferencias y en cómo, una vez comprendidas, traducirlas de forma efectiva en programas y políticas públicas. Como veremos más adelante, estas diferencias en el exceso de peso se expresan también entre beneficiarios y no beneficiarios de programas sociales en el Perú.

El aumento del sobrepeso y la obesidad es motivo de preocupación por diversas razones. En primer lugar, porque, como se ha descrito, afectan a más de la mitad de la población, seguirán aumentando y serán cada vez más comunes en niños y adolescentes. En segundo lugar, porque, como veremos luego, la epidemia se asocia a cambios en los estilos de vida de las poblaciones, cada vez más sedentarias y con dietas que se alejan del patrón alimentario tradicional de cada región, cambios que, con el tiempo, se convierten en hábitos difíciles de modificar, y, en tercer lugar, por supuesto, porque el sobrepeso y la obesidad tienen un enorme impacto en la salud y en la calidad de vida.

El aumento del IMC, incluso desde niveles inferiores al sobrepeso, es un factor que incrementa el riesgo de sufrir ENT, en particular enfermedades cardiovasculares, cáncer, diabetes, osteoartritis y enfermedad renal crónica ${ }^{(1)}$. En el Perú, el Ministerio de Salud ha estimado que un quinto de la carga de enfermedad del año 2011 correspondió a enfermedades asociadas a sobrepeso y obesidad (6), a lo que se suma el que las ENT son la principal causa de mortalidad en el Perú, siendo las enfermedades cardiovasculares las más letales dentro de este grupo ${ }^{(7)}$.

\section{FACTORES ASOCIADOS A LA OBESIDAD: LA IMPORTANCIA DE LA DISPONIBILIDAD Y ACCESO A ALIMENTOS}

El sobrepeso y obesidad son resultado de un desbalance entre la cantidad de calorías que se consumen y las que 
se gastan. Este desbalance se ha profundizado por el cambio en los patrones de alimentación, caracterizado por más alimentos procesados, ricos en azúcares y grasas añadidas ${ }^{(8)} \mathrm{y}$, simultáneamente, por la reducción la actividad física debido a la creciente urbanización, mecanización del trabajo y motorización del transporte ${ }^{(9)}$. En este desequilibrio entre ingesta y gasto de energía, intervienen factores que van desde la variabilidad genética hasta aspectos del entorno, que incluyen, por ejemplo, la cultura, la disponibilidad y acceso a alimentos baratos y de alta palatabilidad, el acceso a espacios para hacer actividad física, la publicidad, y las políticas públicas. La combinación de estos factores puede dar lugar a ambientes obesogénicos, aquellos que favorecen una alta ingesta de energía y poca actividad física, promoviendo la obesidad ${ }^{(9)}$.

PERU MIGRANT (10), un estudio que comparó la salud de pobladores rurales, migrantes de zonas rurales a urbanas y pobladores urbanos, nos da más luces sobre cómo, en el Perú, se expresan en el peso las diferencias del entorno y los estilos de vida. Este estudio del año 2007, encontró grandes diferencias y un gradiente muy claro de obesidad entre los tres grupos: $34,0 \%$ en el urbano, $21,3 \%$ en el migrante, y $3,0 \%$ en el rural. Esto sugiere que el grupo migrante asimiló los hábitos de la vida urbana, lo que se expresaba en un nivel de obesidad similar al de los que siempre habían vivido en la ciudad y muy superior del de aquellos que se habían quedado en el campo.

La disponibilidad y acceso a unos u otros alimentos influirá significativamente en la dieta y el peso. Este acceso puede ser físico -tener alimentos cerca-, económico -a precios accesibles-, nutricional -de alto valor nutricionalo cultural -adecuados a la cultura del consumidor- (11). En los últimos años, las frutas y verduras han subido de precio, mientras que los alimentos y las bebidas menos saludables son más baratos y están disponibles en más lugares y puntos de venta ${ }^{(12)}$, un escenario que restringe la posibilidad de acceder a una alimentación saludable para aquellos en situación de pobreza.

Los cambios en la alimentación han ocurrido de forma global, aunque en algunos grupos con mayor intensidad. En el Perú, entre 2004 y 2009, el consumo de frutas y verduras disminuyó en todos los niveles de riqueza, con un promedio nacional de consumo diario de frutas y de verduras de dos porciones y una porción respectivamente ${ }^{(4)}$. En ese mismo periodo, el consumo de alimentos y bebidas listas para consumir fue el que más aumentó, creciendo también el consumo de alimentos preparados fuera del hogar. Esto refleja cambios importantes y extendidos en los hábitos de alimentación, cada vez más caracterizados por alimentos de fácil preparación o listos para consumir ${ }^{(13)}$
Los pobres son los más sensibles al aumento o reducción de precios al definir su menú diario. Además, dietas más baratas están asociadas a un menor consumo de vegetales, frutas, granos enteros y pescado ${ }^{(14)}$. En el Perú, únicamente $9,3 \%$ de la población consume cinco porciones de frutas y/o verduras al día, pero este consumo recomendado es bastante más infrecuente en zonas rurales $(4,5 \%)$ que urbanas $(12,9 \%)$ así como en el quintil inferior de riqueza $(3,6 \%)$ que en el superior $(19,5 \%)^{(5,15)}$. En buena cuenta, es entre los beneficiarios de programas sociales, donde esperaríamos que los alimentos más consumidos sean fuente de carbohidratos y los menos consumidos sean, entre otros, frutas y verduras.

\section{PROGRAMAS SOCIALES DE COMPLEMENTACIÓN ALIMENTARIA Y OBESIDAD}

Si bien la desnutrición y la obesidad coexisten en el Perú y otros países de América Latina, las políticas públicas suelen centrarse en atender la primera de estas condiciones ${ }^{(16)}$. En el Perú existen programas sociales que buscan complementar la alimentación de las personas en situación de pobreza o vulnerabilidad para contribuir así a la seguridad alimentaria y reducir la desnutrición. En 2014, uno de cada cuatro hogares del Perú tuvo, al menos, a un miembro beneficiario de un programa alimentario, lo que era más frecuente en zonas rurales $(43,8 \%)$ que urbanas $(19,7 \%)^{(17)}$.

Entre los programas sociales de complementación alimentaria más importantes del Perú, se encuentran el Programa Nacional de Alimentación Escolar Qali Warma (en adelante Qali Warma), creado en el año 2013, así como el Programa del Vaso de Leche y el de Comedores Populares, ambos con varias décadas de funcionamiento. La Tabla 2 describe los objetivos, beneficiarios, características y entidades a cargo de estos programas sociales, que, en conjunto, llevan diariamente alimentos a millones de peruanos cuyas condiciones de vida los ponen en riesgo de sufrir de desnutrición, pero también, cada vez más, de sobrepeso y obesidad.

En el caso de los comedores populares, los gobiernos locales entregan ciertos alimentos, fundamentalmente carbohidratos, pero casi nunca frutas y verduras, que son usados para elaborar el menú diario, cuyo diseño y preparación queda a cargo de las mismas cocineras y beneficiarias. Esto asegura un menú adecuado a las preferencias de los comensales, pero no necesariamente a los requerimientos nutricionales de esa población. Tanto el Programa del Vaso de Leche como el Programa Qali Warma cuentan con nutricionistas, aunque las raciones para el desayuno no consideran frutas diariamente y tampoco las frutas 
y verduras están consideradas dentro de los alimentos para el almuerzo. Todo esto invita a reflexionar sobre si los programas sociales de complementación alimentaria garantizan realmente el acceso a dietas balanceadas, cuando están ausentes ciertos grupos de alimentos.

Si bien estos y otros programas sociales han sido creados para combatir la desnutrición y mejorar la salud, existe evidencia en países como México y Chile que indica que los beneficiarios de programas sociales podrían tener más riesgo de sobrepeso y obesidad (18,19). En el Perú, una investigación encontró que las madres que eran usuarias frecuentes del Programa Comedores Populares tenían casi el doble de riesgo de obesidad que las no usuarias ${ }^{(20)}$, y otra reveló una asociación entre ser beneficiaria de algún programa de complementación alimentaria y tener mayor riesgo de sobrepeso y obesidad, aunque solo en mujeres que vivían en hogares que no estaban en situación de pobreza ${ }^{(21)}$. Los resultados de estas investigaciones no han sido del todo negativos, pues el primero de estos estudios reveló que los niños beneficiarios del Programa del Vaso de Leche tenían una reducción de $63 \%$ del riesgo de obesidad ${ }^{(20)}$. La mayor actividad física en los niños en comparación con sus madres podría ser una de las explicaciones de las diferencias, así como el hecho que el Programa Vaso de Leche cuente con nutricionistas para la planificación de raciones.

Tabla 2. Principales programas sociales de complementación alimentaria en el Perú

\begin{tabular}{|c|c|c|c|c|}
\hline Programa & Objetivo & Alimentos entregados & Cobertura & Institución \\
\hline Vaso de Leche & $\begin{array}{l}\text { Mejorar el estado nutricional y } \\
\text { la calidad de vida de los más } \\
\text { pobres a través de la entrega } \\
\text { de leche para los desayunos. } \\
\text { Su primera prioridad son } \\
\text { los niños menores } 6 \text { años, } \\
\text { gestantes y madres que dan } \\
\text { de lactar. El segundo grupo } \\
\text { prioritario son los niños de } 7 \\
\text { a } 13 \text { años, adultos mayores y } \\
\text { pacientes con tuberculosis. }\end{array}$ & $\begin{array}{l}\text { Una ración diaria de } 250 \mathrm{~mL} \\
\text { de leche o algún alimento } \\
\text { equivalente preparado. } \\
\text { En muchos casos, las } \\
\text { raciones no se entregan } \\
\text { diariamente sino semanal o } \\
\text { mensualmente }{ }^{(22)} \text {. }\end{array}$ & $\begin{array}{l}\text { Tres millones de } \\
\text { beneficiarios que } \\
\text { participan en los } \\
66046 \text { Comités } \\
\text { de Vaso de Leche. } \\
\text { Los principales } \\
\text { beneficiarios son los } \\
\text { niños de } 0 \text { a } 6 \text { años } \\
\text { ( } 61,2 \%) \text {, seguido } \\
\text { por los niños de } 7 \\
\text { a } 13 \text { años ( } 18,1 \%) \text {. } \\
\text { Cajamarca es el } \\
\text { departamento } \\
\text { con más número } \\
\text { de comités y } \\
\text { beneficiarios }(7,2 \text { y } \\
6,2 \%) \text {, en segundo } \\
\text { lugar está Piura }(6,7 \\
\text { y } 5,4 \%) \text { y en tercero } \\
\text { Puno (5,9 y } 5,2 \%){ }^{(23)} \text {. }\end{array}$ & $\begin{array}{l}\text { Gobiernos } \\
\text { locales }\end{array}$ \\
\hline $\begin{array}{l}\text { Comedores } \\
\text { Populares }\end{array}$ & $\begin{array}{l}\text { Mejorar el acceso a alimentos } \\
\text { a familias en condición } \\
\text { de pobreza y pobreza } \\
\text { extrema que viven en } \\
\text { áreas periurbanas y rurales } \\
\text { mediante la preparación de } \\
\text { almuerzos a bajo costo. }\end{array}$ & $\begin{array}{l}\text { El paquete de alimentos } \\
\text { consiste en arroz, menestra, } \\
\text { aceite y algún cereal, que } \\
\text { cubren entre } 25 \text { y } 30 \% \text { de } \\
\text { los alimentos que se usan } \\
\text { para preparar las raciones } \\
\text { que venden y distribuyen. Un } \\
\text { grupo de } 1800 \text { comedores } \\
\text { de Lima reciben, además, } \\
\text { un subsidio monetario } \\
\text { equivalente a } 0,25 \text { soles por } \\
\text { ración }{ }^{(24)} \text {. }\end{array}$ & $\begin{array}{l}\text { Existen } 19000 \\
\text { comedores que } \\
\text { benefician alrededor } \\
\text { de } 1,1 \text { millones } \\
\text { de personas en } \\
\text { todo el país. Lima } \\
\text { Metropolitana } \\
\text { concentra la } \\
\text { mayor cantidad de } \\
\text { comedores populares } \\
(16,2 \%) \text { y beneficiarios } \\
(23,2 \%)^{(23)} \text {. }\end{array}$ & $\begin{array}{l}\text { Gobiernos } \\
\text { locales }\end{array}$ \\
\hline $\begin{array}{l}\text { Programa } \\
\text { Nacional de } \\
\text { Alimentación } \\
\text { Escolar Qali } \\
\text { Warma }\end{array}$ & $\begin{array}{l}\text { Mejorar la alimentación de } \\
\text { los niños desde los } 3 \text { años } \\
\text { de edad, matriculados en } \\
\text { instituciones educativas } \\
\text { públicas. }\end{array}$ & $\begin{array}{l}\text { Desayunos y almuerzos } \\
\text { planificados según región } \\
\text { (básicamente cereales y } \\
\text { productos de origen animal), } \\
\text { los cuales son preparados y } \\
\text { distribuidos por los comités } \\
\text { de compra, conformados por } \\
\text { docentes, padres de familia } \\
\text { y la comunidad educativa en } \\
\text { general. }\end{array}$ & $\begin{array}{l}\text { Se han atendido } 3 \\
254363 \text { escolares } \\
\text { pertenecientes a } 57 \\
039 \text { instituciones } \\
\text { educativas de las } 24 \\
\text { regiones del país }{ }^{(25)} \text {. }\end{array}$ & $\begin{array}{l}\text { Ministerio de } \\
\text { Desarrollo e } \\
\text { Inclusión Social }\end{array}$ \\
\hline
\end{tabular}




\section{INVESTIGACIONES EN \\ PROGRAMAS SOCIALES Y ESPACIOS COMUNITARIOS PARA MEJORAR LA DISPONIBILIDAD DE ALIMENTOS: EXPERIENCIAS LOCALES E INTERNACIONALES}

\begin{abstract}
Invitados a reflexionar acerca del rol potencial de los programas sociales en prevenir o reducir la obesidad, creímos interesante resumir unas pocas investigaciones o programas en comedores populares y otros espacios comunitarios, como escuelas, para aumentar la disponibilidad de alimentos y mejorar la salud de usuarios o beneficiarios. Queremos así extraer ideas y lecciones que podrían servir como pauta para diseñar políticas y programas dirigidos a mejorar la alimentación y reducir el impacto de la obesidad en los millones de personas que son beneficiarias y usuarias de los programas sociales, las escuelas públicas y otros espacios comunitarios en el Perú.
\end{abstract}

\section{INTERVENCIONES EN COMEDORES POPULARES}

Entre 1991 y 1993, durante una profunda crisis económica, el Instituto de Investigación Nutricional (IIN) implementó la "La Mejor Compra" (26), proyecto que buscaba aumentar la capacidad y disposición de los comedores populares para utilizar productos nutritivos de bajo costo y mejorar la nutrición de familias pobres de Lima. El proyecto involucró una alianza entre el IIN, 247 comedores, empresas privadas y medios de comunicación. En mercados locales se realizó un monitoreo de precios de los alimentos más utilizados, identificando los que brindaban más energía y proteínas al menor costo. Estos productos se denominaron la mejor compra y se usaron para diseñar recetas nutritivas promovidas en campañas de comunicación. La evaluación evidenció buena recordación y uso de las recetas, favoreciendo la accesibilidad de alimentos nutritivos a familias de bajos ingresos, con estrategias que incorporaban su cultura alimentaria.

En el año 2000, el IIN implementó una intervención comunitaria de nueves meses para mejorar la ingesta y la biodisponibilidad de hierro entre mujeres adolescentes usuarias de comedores de Lima, quienes habitualmente sufrían de anemia. La intervención incluía sesiones educativas y abastecimiento de alimentos altos en hierro, como sangre e hígado de pollo. El estudio, aleatorizado y con comedores control, encontró un aumento mayor de conocimientos sobre la anemia y una mejora en la ingesta de hierro en los comedores intervenidos, aunque sin lograr aumentos significativos en la hemoglobina ${ }^{(27)}$.

"Cocinando para la Salud" fue un proyecto de tres etapas, diseñado e implementado por CRONICAS
Centro de Excelencia en Enfermedades Crónicas entre 2012 y 2014 . Su objetivo era evaluar estrategias para promover la venta y consumo de menús más saludables en comedores populares, como una vía para prevenir las ENT en poblaciones de bajos ingresos. La primera etapa del proyecto, en 2012, fue un estudio cualitativo para conocer la organización y funcionamiento de los comedores. La segunda, en 2013, involucró a 432 usuarias de 48 comedores de Lima, que fueron encuestadas para conocer su disposición para incrementar su gasto si se ofrecieran menús con ensaladas frescas y/o frutas. Se utilizó una metodología denominada modelo de elección discreta, en la que cada participante debía elegir, en ocho oportunidades, entre tres menús que variaban en precio, tipo de productos (por ejemplo: ensalada, sopa, segundo, carne, fruta) y tamaño o unidades de estos productos. Los resultados confirmaron que el precio es lo más importante para las usuarias, pero también demostraron su disposición a invertir una mayor cantidad de dinero, hasta un nuevo sol adicional por menú, si estos incluyeran alimentos saludables como ensaladas o frutas ${ }^{(28)}$.

La tercera etapa de "Cocinando para la Salud", en 2014 , consistió en nueves semanas de ventas de frutas o ensaladas como complementos del menú de ocho comedores de Lima -cuatro ofreciendo frutas, cuatro ensaladas-, a los que sumaban cuatro comedores de control. Previamente, se capacitó a las cocineras y se realizaron campañas de marketing de bajo presupuesto para promover la compra de los complementos, bajo el lema "ponle fruta/ensalada a tu menú". En esta etapa del estudio, aún por publicarse, las mujeres de los ocho comedores vendieron más de 1000 porciones de frutas o ensaladas por semana, lo que demuestra el interés y disposición de los usuarios por adquirirlas, y la factibilidad de prepararlas y ofrecerlas en los comedores populares.

\section{INTERVENCIONES EN COLEGIOS}

Las preferencias alimentarias se forman desde la infancia y se ven influidas por agentes de socialización como los padres, maestros y pares. Eso hace de las escuelas un escenario ideal para formar hábitos de alimentación saludables. Ofrecer frutas y verduras en lugares donde el acceso a alimentos saludables es limitado, como en las escuelas, ha demostrado tener resultados positivos e, incluso, se ha visto que las buenas prácticas de alimentación son reproducidas por los niños en sus hogares ${ }^{(29)}$. Además, las intervenciones que entregan alimentos son más efectivas si se acompañan de educación nutricional ${ }^{(30)}$.

Si bien en el Perú no conocemos de estudios que evalúen el impacto de intervenciones para mejorar la 
disponibilidad de alimentos saludables en la escuela, los resultados son positivos en diversos países, por lo que podría esperarse una situación similar en el Perú. Lo que existe actualmente son intervenciones educativas, de varios gobiernos locales de Lima en escuelas de su distrito, dirigidas a escolares, padres, profesores y expendedores de cafeterías. La Municipalidad de Lima Metropolitana, en concreto, ha establecido el Programa de Escuelas Saludables, que incluye la supervisión de la calidad nutricional de los refrigerios, educación nutricional a padres, madres, tutores y personal docente y el monitoreo del estado nutricional de los escolares de inicial y primaria. El Programa Punto Saludable, de la Municipalidad de Miraflores, realiza actividades similares a las descritas, pero a nivel de primaria y secundaria (31).

\section{INTERVENCIÓN EN UNA PANADERÍA MUNICIPAL}

Durante 2014 se implementó una intervención para evaluar la factibilidad de reducir la cantidad de sal en el 'pan francés' vendido por la panadería de la Municipalidad de Miraflores (32). Una fase inicial del estudio había demostrado que los usuarios de la panadería no distinguían una reducción del $20 \%$ en la cantidad de sal usada al preparar el pan más popular entre los vecinos. Usando un diseño cuasiexperimental, se comparó las ventas de pan de los cinco años previos a la introducción del nuevo pan, con las ventas durante los seis meses de intervención y no se encontraron diferencias. Además, la disminución de 0,10 gramos de sal por pieza de pan, supuso una reducción de 0,36 gramos de sal en la ingesta diaria de cada cliente que, en promedio, consumía 3,6 panes. Estos resultados demuestran la factibilidad de la reducción de la sal en uno de los productos más consumidos del Perú sin afectar su sabor ni las ventas de los panaderos.

\section{PROGRAMAS SOCIALES Y PREVENCIÓN DE LA OBESIDAD EN EL PERÚ: REFLEXIONES FINALES}

El acelerado y significativo aumento del sobrepeso y la obesidad a nivel mundial, ha dejado en claro que se trata de epidemia global que afecta cada vez más duramente a los más pobres. Lamentablemente, no contamos aún con intervenciones eficaces para hacer frente a esta situación a nivel poblacional, por la cual existe una demanda urgente por encontrar nuevas y mejores vías para atenuar su crecimiento.

El mensaje fundamental de esta breve reflexión es que los programas sociales en el Perú, en especial los de complementación alimentaria, podrían ser una plataforma para promover una mejor alimentación entre aquellos ciudadanos que viven en condiciones de pobreza y vulnerabilidad, facilitando el acceso a una canasta de alimentos que permita no solo combatir la desnutrición, sino además reducir la obesidad y enfrentar el avance de las enfermedades crónicas no transmisibles. Se trata de aprovechar, complementar y potenciar programas sociales actualmente establecidos, con una vasta experiencia en la preparación y la distribución de alimentos a nivel nacional, reconocidos y vinculados con una amplia red de organizaciones sociales, y con la capacidad de influir, en el buen sentido, en la alimentación diaria de millones de personas, entre las que se cuentan aquellas en las que, como hemos visto, el sobrepeso y la obesidad son cada vez más frecuentes.

Las investigaciones e intervenciones que repasamos en secciones previas, sin pretender gran exhaustividad, nos dan luces sobre algunas de las muchas estrategias que podrían implementarse en los programas sociales del país para incluir como uno de sus objetivos la reducción de la obesidad. Un tema recurrente en los estudios e importante para frenar la obesidad es mejorar la disponibilidad y el acceso a alimentos adecuados en términos de costos, nutrición y cultura. Varios de estos estudios sugieren la posibilidad de aumentar el consumo de ciertos alimentos en los comedores populares mejorando el acceso. Incluir frutas y ensaladas como complementos del menú del comedor, lo que podría ampliarse a las escuelas de Qali Warma, demostró en el estudio de CRONICAS ser una estrategia bien recibida por las comensales, quienes pagaban por estos complementos.

Esta mejora del acceso a productos esenciales, pero inusuales en programas sociales, como frutas y verduras, podría potenciarse con estrategias como las del proyecto "La Mejor Compra", para detectar los mejores productos y precios del mercado. Esta estrategia podría hacerse masiva, menos costosa y en tiempo real si se utilizara la tecnología disponible, que podría, por ejemplo, permitir que productores o comerciantes ofrezcan los alimentos frescos del día de mejor precio a través de mensajes de texto o aplicativos sencillos. Además, la exitosa experiencia de reducir el sodio en la producción del pan, un producto de consumo masivo, podría extenderse a los desayunos de Qali Warma e incluir, por ejemplo, la adición de ingredientes que hagan más nutritivo a este alimento esencial. A esta inclusión de nuevos y mejores productos, se le podría sumar la reducción, luego de una evaluación, de algunos otros, como ciertas grasas, o la introducción de modos más saludables de cocinar, reduciendo, por ejemplo, las frituras de los comedores populares.

Otras estrategias que podrían implementarse, en base siempre a la información revisada, es el uso de componentes de educación e información para mejorar 
la alimentación de los beneficiarios de los programas sociales, con énfasis en la educación de los niños, así como el desarrollo de campañas culturalmente apropiadas, enfocadas en los programas sociales, para educar y promover cambios en hábitos de consumo. Conocer la cultura alimentaria de la región donde se trabaja y tomarla como punto de partida para construir cualquier estrategia, lo que entendemos se haría en Qali Warma, es otra buena práctica, como lo es también la activa inclusión de las personas beneficiarias o usuarias en la construcción de las soluciones a implementar, para garantizar así sentido de apropiación, compromiso y una adecuada inclusión de sus voces y miradas.

La evaluación nutricional de los alimentos y platos ofrecidos por los programas sociales es clave. La presencia de profesionales de nutrición en Qali Warma y en el Programa del Vaso de Leche debería extenderse y acercarse a las mujeres de los comedores populares, no solo para la elección de productos y el diseño de los menús, sino también para ofrecer asesoría en la adecuada manipulación y cocción de los alimentos.

Transformar los programas sociales de complementación alimentaria en una de las puntas de lanza para enfrentar la obesidad en los barrios y las comunidades pobres del Perú, no sería, sin duda, una lucha que esté libre de viejas y nuevas dificultades, ni la solución definitiva a un problema que requiere respuestas múltiples. A la filtración e inadecuada focalización, la corrupción o el manejo populista de los programas sociales, se sumarían nuevos retos como, por ejemplo, la adquisición y manipulación segura de productos como frutas y verduras, o las naturales resistencias a reducir las frituras, de gran popularidad en los paladares locales, o incluir ensaladas, menos apetecibles que las anteriores.

Más allá de las dificultades, que es importante identificar, creemos que los programas sociales tienen el potencial de ser un espacio idóneo para reducir la obesidad en el Perú, una idea que esta breve reflexión no pretende agotar, sino que entregamos más bien como una invitación para abrir el diálogo y explorar, con más y mejor evidencia, la posibilidad de hacerla realidad.

Fuentes de financiamiento: autofinanciado.

Conflictos de interés: los autores declaran no tener conflictos de interés.

Contribución de autoría: FDC y LSG concibieron el artículo de manera conjunta, realizaron la revisión de la literatura, participaron en su redacción y aprobaron su versión final.

\section{REFERENCIAS BIBLIOGRÁFICAS}

1. Popkin BM, Adair LS, Ng SW. The Global nutrition transition: the pandemic of obesity in developing countries. Nutr Rev. 2012;70(1):321. doi: 10.1111/j.17534887.2011.00456.x

2. $\mathrm{Ng} \mathrm{M}$, Fleming $\mathrm{T}$, Robinson $\mathrm{M}$, Thomson B, Graetz N, Margono C, et al. Global, regional, and national prevalence of overweight and obesity in children and adults during 19802013: a systematic analysis for the Global Burden of Disease Study 2013. Lancet. 2014;384(9945):766-81. doi: 10.1016/S0140-6736(14)60460-8

3. Popkin BM, Slining MM. New dynamics in global obesity facing low- and middle-income countries. Obes Rev. 2013;14 Suppl 2:11-20. doi:10.1111/obr.12102

4. Instituto Nacional de Estadística e Informática. Perú: Enfermedades No Transmisibles y Transmisibles, 2015 [Internet]. Lima: INEI; 2016 [citado 14 feb 2017]. Disponible en: https://www.inei.gob.pe/media/
MenuRecursivo/publicaciones digitales/Est/Lib1357/index.html

5. Factores de riesgo conductuales para enfermedades crónicas no transmisibles [Internet]. Lima: CENAN - INS; 2015 [citado el 20 de junio de 2016]. Disponible en: http:// www.observateperu.ins.gob.pe/salasituacional/situacion-nutricional

6. Ministerio de Salud del Perú. Un gordo problema: sobrepeso y obesidad en el Perú. Lima:MINSA; 2012.

7. Huicho L, Trelles M, Gonzales F, Mendoza W, Miranda J. Mortality profiles in a country facing epidemiological transition: an analysis of registered data. BMC Public Health. 2009;9:47. doi: 10.1186/1471-2458$9-47$

8. Sun J, Buys NJ, Hills AP. Dietary pattern and its association with the prevalence of obesity, hypertension and other cardiovascular risk factors among Chinese older adults. Int J Environ Res Public Health. 2014;11(4):3956-71. doi: $10.3390 /$ ijerph 110403956
9. Swinburn BA, Sacks G, Hall KD, McPherson K, Finegood DT, Moodie ML, et al. The global obesity pandemic: shaped by global drivers and local environments. Lancet. 2011;378(9793):804-14. doi: 10.1016/S0140-6736(11)60813-1

10. Miranda JJ, Gilman RH, Garcia HH, Smeeth L. The effect on cardiovascular risk factors of migration from rural to urban areas in Peru: PERU MIGRANT Study. BMC Cardiovasc Disord. 2009;9:23. doi: 10.1186/1471-2261-9-23

11. Gittelsohn J, Sharma S. Physical, consumer, and social aspects of measuring the food environment amongdiverselow-income populations. American journal of preventive medicine. 2009;36(4 Suppl):S161-5. doi: 10.1016/j.amepre.2009.01.007

12. Popkin BM. Agricultural policies, food and public health. EMBO Rep. 2011;12(1):11-8. doi: 10.1038/ embor.2010.200

13. Díaz R. Análisis económico de la ingesta de alimentos en el Perú. Lima: IEP; 2010. 
14. Rehm CD, Monsivais P, Drewnowski A. Relation between diet cost and Healthy Eating Index 2010 scores among adults in the United States 2007-2010. Prev Med. 2015;73:70-5. doi: 10.1016/j.ypmed.2015.01.019

15. Consumo de frutas y verduras por día [Internet]. Lima: CENAN INS; 2016 [citado el 21 de junio de 2016]. Disponible en: http://www. observateperu.ins.gob.pe/images/ archivos/factores-de-riesgo/1.2Consumo-de-frutas.pdf.

16. Rivera JÁ, de Cossío TG, Pedraza LS, Aburto TC, Sánchez TG, Martorell R. Childhood and adolescent overweight and obesity in Latin America: a systematic review. Lancet Diabetes Endocrinol. 2014;2(4):321-32. doi: $10.1016 /$ S2213-8587(13)70173-6

17. Instituto Nacional de Estadística e Informática. Encuesta Nacional de Hogares 2014. Lima: INEI; 2015

18. Fernald LC, Gertler PJ, Neufeld LM. Role of cash in conditional cash transfer programmes for child health, growth, and development: an analysis of Mexico's Oportunidades. Lancet. 2008;371(9615):828-37. doi: 10.1016/S0140-6736(08)60382-7

19. Corvalan C, Uauy R, Flores R, Kleinbaum D, Martorell R. Reductions in the energy content of meals served in the Chilean National Nursery School Council Program did not consistently decrease obesity among beneficiaries. J Nutr. 2008;138(11):2237-43.

20. Carrillo-Larco RM, Miranda JJ, Bernabe-Ortiz A. Impact of food assistance programs on obesity in mothers and children: a prospective cohort study in Peru. Am J Public Health. 2016;106(7):1301-7. doi: 10.2105/AJPH.2016.303191

21. Chaparro MP, Bernabe-Ortiz A, Harrison GG. Association between food assistance program participation and overweight. Revista de Saúde Pública. 2014;48(6):889-98. doi: 10.1590/S0034-8910.2014048005359

22. Alcázar L, López-Cálix JR, Wachtenheim E. Las pérdidas en el camino, fugas en el gasto público: transferencias municipales, vaso de leche y sector educación. . Lima: Instituto Apoyo; 2003.

23. Instituto Nacional de Estadística e Informática. Estadísticas Municipales 2013. Lima: INEI, 2014.

24. Alcázar L. ¿Por qué no funcionan los programas alimentarios y nutricionales en el Perú?: riesgos y oportunidades para su reforma. En: Grupo de análisis para el desarrollo, editores. Investigación, políticas y desarrollo en el Perú. Lima:GRADE; 2007. 185234.

25. INFOMIDIS [Internet]. Lima:MIDIS; 2016 [citado el 21 de junio de 2016]. Disponible en: http:// www.midis.gob.pe/mapas/infomidis

26. Organización Panamericana de la Salud. Nutrition and an active life: from knowledge to action. Freire WB, editora. Washington, D.C.: OPS; 2005.

27. Creed-Kanashiro HM, Uribe TG, Bartolini RM, Fukumoto MN, Lopez TT, Zavaleta NM, et al. Improving dietary intake to prevent anemia in adolescent girls through community kitchens in a periurban population of Lima, Peru. J Nutr. 2000;130(2S Suppl):459S-61S.

28. Buttorff C, Trujillo AJ, Diez-Canseco F, Bernabe-Ortiz A, Miranda JJ. Evaluating consumer preferences for healthy eating from Community Kitchens in low-income urban areas: A discrete choice experiment of Comedores Populares in Peru. Soc Sci
Med. 2015;140:1-8. doi: 10.1016/j. socscimed.2015.06.033

29. Sharma S, Helfman L, Albus K, Pomeroy M, Chuang RJ, Markham C. Feasibility and acceptability of brighter bites: a food co-op in schools to increase access, continuity and education of fruits and vegetables among low-income populations. J Prim Prev. 2015;36(4):281-6. doi: 10.1007/ s10935-015-0395-2

30. Evans CE, Christian MS, Cleghorn CL, Greenwood DC, Cade JE. Systematic review and meta-analysis of school-based interventions to improve daily fruit and vegetable intake in children aged 5 to $12 \mathrm{y}$. Am J Clin Nutr. 2012;96(4):889-901. doi: $10.3945 /$ ajcn.111.030270

31. Centro Nacional de Alimentación y Nutrición. Iniciativa de los Municipios contra el sobrepeso y obesidad [Internet]. Lima: CENAN - INS; 2015 [citado el 14 de febrero de 2017]. Disponible en: http:// www.observateperu.ins.gob.pe/ municipalidades

32. Saavedra-Garcia L, Sosa-Zevallos V, Diez-Canseco F, Miranda JJ, BernabeOrtiz A. Reducing salt in bread: a quasi-experimental feasibility study in a bakery in Lima, Peru. Public Health Nutr. 2016;19(6):976-82. doi: $10.1017 /$ S1368980015001597

Correspondencia: Francisco Diez Canseco Montero

Dirección: Av. Armendáriz 497, 2. ${ }^{\circ}$ piso,

Miraflores. Lima, Perú.

Teléfono: (+511) 2416978

Correoelectrónico:fdiezcanseco@upch.pe

Nuestros artículos se encuentran indizados en:

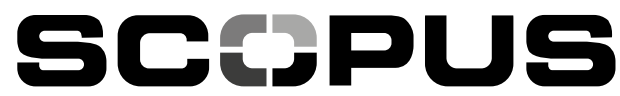

wWW.scopus.com 